\title{
Existence of positive solutions to negative power nonlinear integral equations with weights
}

\author{
Hang Chen', Qianqiao Guo ${ }^{1 *}$ and Qian Wang
}

\section{"Correspondence:}

gqianqiao@nwpu.edu.cn

'School of Mathematics and

Statistics, Northwestern

Polytechnical University, Xi'an, China

\begin{abstract}
This paper is devoted to the existence and non-existence of positive solutions to the following negative power nonlinear integral equation related to the sharp reversed Hardy-Littlewood-Sobolev inequality:

$$
f^{q-1}(x)=\int_{\Omega} \frac{K(x) f(y) K(y)}{|x-y|^{n-\alpha}} d y+\lambda \int_{\Omega} \frac{G(x) f(y) G(y)}{|x-y|^{n-\alpha-\beta}} d y, \quad f \geq 0, x \in \bar{\Omega},
$$

where $0<q<1, \alpha>n, 0<\beta<\alpha-n, \lambda \in \mathbb{R}, \Omega$ is a smooth bounded domain, $K(x)$, $G(x)$ are positive continuous functions in $\bar{\Omega}$. For $K \equiv G \equiv 1$, the existence and non-existence of positive solutions to the equation have been studied by Dou-Guo-Zhu (2019). In this paper we consider the existence and non-existence of positive solutions to the above integral equation with the general weight functions $K(x), G(x)$.
\end{abstract}

MSC: 45G05; 35A15; 35B44

Keywords: Reversed Sharp Hardy-Littlewood-Sobolev inequality; Positive solution; Integral equation

\section{Introduction}

In this paper we consider the existence and non-existence of positive solutions to the following negative power nonlinear integral equation:

$$
f^{q-1}(x)=\int_{\Omega} \frac{K(x) f(y) K(y)}{|x-y|^{n-\alpha}} d y+\lambda \int_{\Omega} \frac{G(x) f(y) G(y)}{|x-y|^{n-\alpha-\beta}} d y, \quad f \geq 0, x \in \bar{\Omega},
$$

where $0<q<1, \alpha>n, 0<\beta<\alpha-n, \lambda \in \mathbb{R}, \Omega$ is a smooth bounded domain, $K(x), G(x)$ are positive continuous functions in $\bar{\Omega}$.

For $0<\alpha<n, G(x) \equiv 1$, the existence and non-existence of positive solutions to (1.1) were studied by Dou-Zhu [2] and Guo-Wang [3] recently. Notice that when $0<\alpha<n$ this nonlinear integral equation is closely related to the sharp Hardy-Littlewood-Sobolev (HLS for short) inequality [4-7].

(c) The Author(s) 2020. This article is licensed under a Creative Commons Attribution 4.0 International License, which permits use, sharing, adaptation, distribution and reproduction in any medium or format, as long as you give appropriate credit to the original author(s) and the source, provide a link to the Creative Commons licence, and indicate if changes were made. The images or other third party material in this article are included in the article's Creative Commons licence, unless indicated otherwise in a credit line to the material. If material is not included in the article's Creative Commons licence and your intended use is not permitted by statutory regulation or exceeds the permitted use, you will need to obtain permission directly from the copyright holder. To view a copy of this licence, visit http://creativecommons.org/licenses/by/4.0/. 
For $\alpha>n$, the existence and non-existence of positive solutions to (1.1) are also studied by Dou-Guo-Zhu [1] when $K(x) \equiv G(x) \equiv 1$. In this case the nonlinear integral equation is related to the sharp reversed HLS inequality obtained by Beckner [8] and Dou-Zhu [9], respectively. In fact, Eq. (1.1) (when $K(x) \equiv 1, \lambda=0$ ) can be seen as the Euler-Lagrange equation of the following minimizing problem related to the reversed HLS inequality:

$$
\xi_{\alpha}(\Omega)=\inf _{f \in L^{\frac{2 n}{n+\alpha}}(\Omega), f \geq 0, f \neq 0} \frac{\int_{\Omega} \int_{\Omega} f(x)|x-y|^{-(n-\alpha)} f(y) d x d y}{\|f\|_{L^{\frac{2 n}{n+\alpha}}(\Omega)}} .
$$

On the other hand, for Eq. (1.1) with $K(x) \equiv 1$ and $\lambda=0$, the blowup behavior of energy maximizing positive solutions as $q \rightarrow\left(\frac{2 n}{n+\alpha}\right)^{+}$when $1<\alpha<n$, and the blowup behavior of energy minimizing positive solution as $q \rightarrow\left(\frac{2 n}{n+\alpha}\right)^{-}$when $\alpha>n$ are also analyzed by Guo [10].

In this paper we consider the integral equation (1.1) for general weight functions $K(x)$, $G(x)$ and $\alpha>n$.

The following condition is needed.

$(\mathcal{T}) . K\left(x_{*}\right)-K(x)=o\left(\left|x-x_{*}\right|^{\gamma}\right)$ as $x \rightarrow x_{*}$, where $K\left(x_{*}\right)=\min _{x \in \bar{\Omega}} K(x), \gamma>0$.

Denote $G\left(\tilde{x}_{*}\right)=\max _{x \in \bar{\Omega}} G(x)$.

The main results are stated as follows.

Theorem 1.1 Assume $\alpha>n, \beta \in(0, \alpha-n), \Omega$ is a smooth bounded domain of diameter $d(\Omega)$.

(i) For $0<q<\frac{2 n}{n+\alpha}$ (subcritical case), $-\frac{K^{2}\left(x_{*}\right)}{d^{\beta}(\Omega) G^{2}\left(\tilde{x}_{*}\right)}<\lambda$, the positive functions $K(x), G(x) \in C^{1}(\bar{\Omega})$, then there is a positive solution $f \in C^{1}(\bar{\Omega})$ to Eq. (1.1).

(ii) For $q=\frac{2 n}{n+\alpha}$ (critical case), $-\frac{K^{2}\left(x_{*}\right)}{d^{\beta}(\Omega) G^{2}\left(\tilde{x}_{*}\right)}<\lambda<0$, the positive functions $K(x), G(x) \in C^{1}(\bar{\Omega})$, assume further that $\beta<n$ and $(\mathcal{T})$ holds, then there is a positive solution $f \in C^{1}(\bar{\Omega})$ to Eq. (1.1).

(iii) For $\frac{2 n}{n+\alpha} \leq q<1$ (critical case and supercritical case), $\lambda \geq 0$, the nonnegative functions $K(x), G(x) \in C^{1}(\bar{\Omega})$, if $\Omega$ is a star-shaped domain with respect to $\tilde{x}$, $(x-\tilde{x}, \nabla K(x)) \geq 0$ and $(x-\tilde{x}, \nabla G(x)) \geq 0$, then there is not any positive $C^{1}(\bar{\Omega})$ solution to Eq. (1.1).

We use $c, C$ throughout the paper to represent positive constants, which may vary from line to line.

\section{Preliminaries}

For simplicity, we denote $p_{\alpha}:=\frac{2 n}{n-\alpha}, q_{\alpha}:=\frac{2 n}{n+\alpha}$ throughout the paper. For $0<q<1$, we also denote $L^{q}(\Omega):=\left\{\left.f\left|\int_{\Omega}\right| f\right|^{q}(x) d x<\infty\right\}$ for any domain $\Omega \subset \mathbb{R}^{n}, L_{+}^{q}(\Omega):=\left\{f \in L^{q}(\Omega) \backslash\{0\}\right.$ : $f \geq 0\}$ and define $\|f\|_{L^{q}(\Omega)}:=\left(\int_{\Omega}|f|^{q}(x) d x\right)^{\frac{1}{q}}$ for $f \in L^{q}(\Omega)$. Notice that $\|f\|_{L^{q}(\Omega)}$ is not a norm if $0<q<1$.

We first recall the sharp reversed HLS inequality on $\mathbb{R}^{n}$.

Theorem A (see $[8,9])$ Let $\alpha>n$. Then

$$
\left|\int_{\mathbb{R}^{n}} \int_{\mathbb{R}^{n}} f(x)\right| x-\left.y\right|^{-(n-\alpha)} g(y) d x d y \mid \geq N_{\alpha}\|f\|_{L^{q_{\alpha}\left(\mathbb{R}^{n}\right)}}\|g\|_{L^{q_{\alpha}\left(\mathbb{R}^{n}\right)}}
$$


for all $f, g \in L^{q_{\alpha}}\left(\mathbb{R}^{n}\right)$, where $N_{\alpha}:=\pi^{\frac{n-\alpha}{2}} \frac{\Gamma\left(\frac{\alpha}{2}\right)}{\Gamma\left(\frac{n}{2}+\frac{\alpha}{2}\right)}\left(\frac{\Gamma\left(\frac{n}{2}\right)}{\Gamma(n)}\right)^{-\frac{\alpha}{n}}$. Moreover, the equality holds if and only if $f(x)=c_{1} g(x)=c_{2}\left(\frac{1}{c_{3}+\left|x-x_{0}\right|^{2}}\right)^{\frac{n+\alpha}{2}}$, where $c_{1}, c_{2}, c_{3}$ are any constants, $x_{0} \in \mathbb{R}^{n}$.

\section{Proofs of the main results}

Here and hereafter we always assume $\alpha>n$.

\subsection{Existence-subcritical case}

We first prove the existence of positive solution to Eq. (1.1) in the subcritical case $0<q<$ $q_{\alpha}$. The following lemma from [2] is needed.

Lemma 3.1 (see [2]) Let $q \in\left(0, q_{\alpha}\right)$. There exists a positive constant $C(n, q, \alpha, \Omega)>0$ such that

$$
\int_{\Omega} \int_{\Omega} f(x)|x-y|^{-(n-\alpha)} f(y) d x d y \geq C(n, q, \alpha, \Omega)\|f\|_{L^{q}(\Omega)}^{2}
$$

for any nonnegative function $f \in L^{q}(\Omega)$.

Now we prove the following lemma, which implies the existence result of part (i) in Theorem 1.1.

Lemma 3.2 Assume the positive functions $K(x), G(x) \in C^{1}(\bar{\Omega})$. Then, for $\left.0<q<q_{\alpha}, \lambda\right\rangle$ $-\frac{K^{2}\left(x_{*}\right)}{d^{\beta}(\Omega) G^{2}\left(\tilde{x}_{*}\right)}$, the infimum

$$
\begin{aligned}
& Q_{\lambda, q}(\Omega) \\
& \quad:=\inf _{f \in L_{+}^{q}(\Omega)} \frac{\int_{\Omega} \int_{\Omega} f(x)\left(K(x)|x-y|^{-(n-\alpha)} K(y)+\lambda G(x)|x-y|^{-(n-\alpha-\beta)} G(y)\right) f(y) d x d y}{\|f\|_{L^{q}(\Omega)}^{2}}
\end{aligned}
$$

is attained by some nonnegative function in $L_{+}^{q}(\Omega)$.

Proof Notice that $\lambda>-\frac{K^{2}\left(x_{*}\right)}{d^{\beta}(\Omega) G^{2}\left(\tilde{x}_{*}\right)}$ and

$$
K(x) K(y)+\lambda G(x)|x-y|^{\beta} G(y) \geq K^{2}\left(x_{*}\right)+\lambda d^{\beta}(\Omega) G^{2}\left(\tilde{x}_{*}\right)>0, \quad x, y \in \Omega .
$$

Then by Lemma 3.1, $Q_{\lambda, q}(\Omega)>0$.

Now we can choose the minimizing positive sequence $\left\{f_{j}\right\}_{j=1}^{\infty}$ in $L_{+}^{q}(\Omega)$ and argue as Lemma 3.2 in [1]. We sketch it for the reader's convenience. Assume $f_{j} \in L^{q_{\alpha}}(\Omega)$ and $\left\|f_{j}\right\|_{L^{q_{\alpha}(\Omega)}}=1$. Then, up to a subsequence,

$$
f_{j}^{q} \rightarrow f_{*}^{q} \quad \text { in } L^{\frac{q \alpha}{q}}(\Omega), \text { as } j \rightarrow \infty
$$

and

$$
\int_{\Omega} f_{j}^{q} \rightarrow \int_{\Omega} f_{*}^{q}, \quad \text { as } j \rightarrow \infty .
$$

As in [1], we have $\left\|f_{j}\right\|_{L^{1}(\Omega)} \leq C$. Thus $\int_{\Omega} f_{*}^{q}>C>0$ via an interpolation inequality and $f_{j}^{q} \rightarrow f_{*}^{q}$ weakly in $L^{\frac{1}{q}}(\Omega)$. For any fixed $x \in \bar{\Omega}, f_{*}^{1-q}(y)|x-y|^{\alpha-n}(K(x) K(y)+\lambda G(x) \mid x-$ 


$$
\begin{aligned}
& \left.\left.y\right|^{\beta} G(y)\right) \in L^{\frac{1}{1-q}}(\Omega) \text {. Therefore } \\
& \qquad \int_{\Omega} f_{j}^{q}(y) f_{*}^{1-q}(y)|x-y|^{\alpha-n}\left(K(x) K(y)+\lambda G(x)|x-y|^{\beta} G(y)\right) d y \\
& \quad \rightarrow \int_{\Omega} f_{*}(y)|x-y|^{\alpha-n}\left(K(x) K(y)+\lambda G(x)|x-y|^{\beta} G(y)\right) d y, \quad \text { as } j \rightarrow \infty .
\end{aligned}
$$

Now we prove that the convergence is uniform for all $x \in \bar{\Omega}$. Firstly, as Lemma 3.2 in [1], we have $\int_{\Omega} f_{j}^{q}(y) f_{*}^{1-q}(y)|x-y|^{\alpha-n}\left(K(x) K(y)+\lambda G(x)|x-y|^{\beta} G(y)\right) d y$ is uniformly bounded for $x \in \bar{\Omega}$. Now it is left to prove that $\int_{\Omega} f_{j}^{q}(y) f_{*}^{1-q}(y)|x-y|^{\alpha-n}\left(K(x) K(y)+\lambda G(x)|x-y|^{\beta} G(y)\right) d y$ is equicontinuous in $\bar{\Omega}$. Notice that $K(x), G(x) \in C^{1}(\bar{\Omega})$ and for any $x_{1}, x_{2}, y \in \bar{\Omega}$,

$$
|| x_{1}-\left.y\right|^{\alpha-n}-\left|x_{2}-y\right|^{\alpha-n} \mid \leq \begin{cases}C\left|x_{1}-x_{2}\right|^{\alpha-n}, & 0<\alpha-n \leq 1, \\ C\left|x_{1}-x_{2}\right|, & \alpha-n>1 .\end{cases}
$$

Then since $\int_{\Omega} f_{j}^{q}(y) f_{*}^{1-q}(y) K(y) d y$ is bounded, for any $x_{1}, x_{2} \in \bar{\Omega}$,

$$
\begin{aligned}
& \left|\int_{\Omega} f_{j}^{q}(y) f_{*}^{1-q}(y)\right| x_{1}-\left.y\right|^{\alpha-n} K(y) d y-\int_{\Omega} f_{j}^{q}(y) f_{*}^{1-q}(y)\left|x_{2}-y\right|^{\alpha-n} K(y) d y \mid \\
& \quad \leq \int_{\Omega} f_{j}^{q}(y) f_{*}^{1-q}(y) K(y)|| x_{1}-\left.y\right|^{\alpha-n}-\left|x_{2}-y\right|^{\alpha-n} \mid d y \\
& \quad \leq C \max \left(\left|x_{1}-x_{2}\right|^{\alpha-n},\left|x_{1}-x_{2}\right|\right) .
\end{aligned}
$$

So $\int_{\Omega} f_{j}^{q}(y) f_{*}^{1-q}(y)|x-y|^{\alpha-n} K(y) d y$ and, by a similar argument, $\lambda \int_{\Omega} f_{j}^{q}(y) f_{*}^{1-q}(y) \mid x-$ $\left.y\right|^{\alpha+\beta-n} G(y) d y$ are equicontinuous in $x \in \bar{\Omega}$. Thus we see that $\int_{\Omega} f_{j}^{q}(y) f_{*}^{1-q}(y)|x-y|^{\alpha-n} \times$ $\left(K(x) K(y)+\lambda G(x)|x-y|^{\beta} G(y)\right) d y$ is equicontinuous in $\bar{\Omega}$.

Now similar to Lemma 3.2 in [1], we can prove

$$
\begin{gathered}
\liminf _{j \rightarrow \infty} \int_{\Omega} \int_{\Omega} f_{j}(x)|x-y|^{\alpha-n}\left(K(x) K(y)+\lambda G(x)|x-y|^{\beta} G(y)\right) f_{j}(y) d x d y \\
\geq \int_{\Omega} \int_{\Omega} f_{*}(x)|x-y|^{\alpha-n}\left(K(x) K(y)+\lambda G(x)|x-y|^{\beta} G(y)\right) f_{*}(y) d x d y .
\end{gathered}
$$

By $\left\|f_{j}\right\|_{L^{q}(\Omega)} \rightarrow\left\|f_{*}\right\|_{L^{q}(\Omega)}>0$ and the above,

$$
\begin{array}{r}
\liminf _{j \rightarrow \infty} \frac{\int_{\Omega} \int_{\Omega} f_{j}(x)|x-y|^{\alpha-n}\left(K(x) K(y)+\lambda G(x)|x-y|^{\beta} G(y)\right) f_{j}(y) d x d y}{\left\|f_{j}\right\|_{L^{q}(\Omega)}^{2}} \\
\geq \frac{\int_{\Omega} \int_{\Omega} f_{*}(x)|x-y|^{\alpha-n}\left(K(x) K(y)+\lambda G(x)|x-y|^{\beta} G(y)\right) f_{*}(y) d x d y}{\left\|f_{*}\right\|_{L^{q}(\Omega)}^{2}} .
\end{array}
$$

That is, $f_{*}$ is the minimizer.

Again as that in [1], we obtain $u \in C^{1}(\bar{\Omega})$. Thus we complete the proof of Theorem 1.1 (i).

Remark 3.3 We assume $\lambda>-\frac{K^{2}\left(\left(_{*}\right)\right.}{d^{\beta}(\Omega) G^{2}\left(\tilde{x}_{*}\right)}$ here to make sure that $Q_{\lambda, q}(\Omega)$ is positive. 


\subsection{Existence-critical case}

Now we establish the existence and the regularity results for the weak solution to (1.1) with critical exponent for $\lambda<0$. Consider

$$
\begin{aligned}
& Q_{\lambda, q_{\alpha}}(\Omega) \\
& \quad=\inf _{f \in L_{+}^{q_{\alpha}}(\Omega)} \frac{\int_{\Omega} \int_{\Omega} f(x)\left(K(x)|x-y|^{-(n-\alpha)} K(y)+\lambda G(x)|x-y|^{-(n-\alpha-\beta)} G(y)\right) f(y) d y d x}{\|f\|_{L^{q_{\alpha}(\Omega)}}^{2}} .
\end{aligned}
$$

Notice that the corresponding Euler-Lagrange equation for extremal functions, up to a constant multiplier, is the integral equation (1.1) with $q=q_{\alpha}$.

We first show the following lemma.

Lemma 3.4 Assume that the positive functions $K(x), G(x) \in C^{1}(\bar{\Omega})$ and $(\mathcal{T})$ holds. Then $Q_{\lambda, q_{\alpha}}(\Omega)<K^{2}\left(x_{*}\right) N_{\alpha}$ for all $\lambda<0$. Further, $0<Q_{\lambda, q_{\alpha}}(\Omega)<K^{2}\left(x_{*}\right) N_{\alpha}$ for any $\lambda \in$ $\left(-\frac{K^{2}\left(x_{*}\right)}{d^{\beta}(\Omega) G^{2}\left(\tilde{x}_{*}\right)}, 0\right)$, where $\beta>0$.

Proof We distinguish two cases: (I) $x_{*} \in \Omega$; (II) $x_{*} \in \partial \Omega$.

(I) Let $x_{*} \in \Omega$. By $(\mathcal{T})$, there exists small $R>0$ such that $K(x)-K\left(x_{*}\right) \leq c\left|x-x_{*}\right|^{\gamma}$ when $x \in B_{R}\left(x_{*}\right) \subset \Omega$. For small $\epsilon>0$, we define

$$
\tilde{f}_{\epsilon}(x)= \begin{cases}f_{\epsilon}(x), & x \in B_{R}\left(x_{*}\right) \subset \Omega, \\ 0, & x \in \mathbb{R}^{n} \backslash B_{R}\left(x_{*}\right),\end{cases}
$$

where $f_{\epsilon}(x)=\epsilon^{-\frac{n+\alpha}{2}} f_{1}\left(\frac{x-x_{*}}{\epsilon}\right)=\left(\frac{\epsilon}{\epsilon^{2}+\left|x-x_{*}\right|^{2}}\right)^{\frac{n+\alpha}{2}}, f_{1}(x)=\left(\frac{1}{1+|x|^{2}}\right)^{\frac{n+\alpha}{2}}$. Notice that $f_{1}$ and its conformal equivalent class $f_{\epsilon}$ are the extremal functions to the sharp reversed HLS inequality (2.1). Obviously, $\tilde{f}_{\epsilon} \in L^{q_{\alpha}}\left(\mathbb{R}^{n}\right)$. By $(\mathcal{T})$, we have

$$
\begin{aligned}
& \int_{\Omega} \int_{\Omega}\left(\frac{K(x) K(y)}{|x-y|^{n-\alpha}}+\frac{\lambda G(x) G(y)}{|x-y|^{n-\alpha-\beta}}\right) \tilde{f}_{\epsilon}(x) \tilde{f}_{\epsilon}(y) d x d y \\
& =\int_{B_{R}\left(x_{*}\right)} \int_{B_{R}\left(x_{*}\right)}\left(\frac{K(x) K(y)}{|x-y|^{n-\alpha}}+\frac{\lambda G(x) G(y)}{|x-y|^{n-\alpha-\beta}}\right) f_{\epsilon}(x) f_{\epsilon}(y) d x d y \\
& \leq \int_{B_{R}\left(x_{*}\right)} \int_{B_{R}\left(x_{*}\right)} \frac{\left(K\left(x_{*}\right)+c\left|x-x_{*}\right|^{\gamma}\right)\left(K\left(x_{*}\right)+c\left|y-x_{*}\right|^{\gamma}\right)}{|x-y|^{n-\alpha}} f_{\epsilon}(x) f_{\epsilon}(y) d x d y \\
& +\lambda \int_{B_{R}\left(x_{*}\right)} \int_{B_{R}\left(x_{*}\right)} \frac{G(x) G(y) f_{\epsilon}(x) f_{\epsilon}(y)}{|x-y|^{n-\alpha-\beta}} d x d y \\
& =\int_{B_{R}\left(x_{*}\right)} \int_{B_{R}\left(x_{*}\right)} \frac{K^{2}\left(x_{*}\right)+K\left(x_{*}\right)\left(c\left|x-x_{*}\right|^{\gamma}+c\left|y-x_{*}\right|^{\gamma}\right)+c^{2}\left|x-x_{*}\right|^{\gamma}\left|y-x_{*}\right|^{\gamma}}{|x-y|^{n-\alpha}} \\
& \times f_{\epsilon}(x) f_{\epsilon}(y) d x d y \\
& +\lambda \int_{B_{R}\left(x_{*}\right)} \int_{B_{R}\left(x_{*}\right)} \frac{G(x) G(y) f_{\epsilon}(x) f_{\epsilon}(y)}{|x-y|^{n-\alpha-\beta}} d x d y \\
& \leq \int_{\mathbb{R}^{n}} \int_{\mathbb{R}^{n}} \frac{K^{2}\left(x_{*}\right) f_{\epsilon}(x) f_{\epsilon}(y)}{|x-y|^{n-\alpha}} d x d y+\lambda \int_{B_{R}\left(x_{*}\right)} \int_{B_{R}\left(x_{*}\right)} \frac{G(x) G(y) f_{\epsilon}(x) f_{\epsilon}(y)}{|x-y|^{n-\alpha-\beta}} d x d y \\
& +c^{2} \int_{B_{R}\left(x_{*}\right)} \int_{B_{R}\left(x_{*}\right)} \frac{\left|x-x_{*}\right|^{\gamma}\left|y-x_{*}\right|^{\gamma} f_{\epsilon}(x) f_{\epsilon}(y)}{|x-y|^{n-\alpha}} d x d y
\end{aligned}
$$




$$
\begin{aligned}
& +2 c \int_{B_{R}\left(x_{*}\right)} \int_{B_{R}\left(x_{*}\right)} \frac{K\left(x_{*}\right)\left|x-x_{*}\right|^{\gamma} f_{\epsilon}(x) f_{\epsilon}(y)}{|x-y|^{n-\alpha}} d x d y \\
= & K^{2}\left(x_{*}\right) N_{\alpha}\left\|f_{\epsilon}\right\|_{L^{q_{\alpha}\left(\mathbb{R}^{n}\right)}}+I_{1}+I_{2}+I_{3},
\end{aligned}
$$

where

$$
\begin{aligned}
& I_{1}:=\lambda \int_{B_{R}\left(x_{*}\right)} \int_{B_{R}\left(x_{*}\right)} \frac{G(x) G(y) f_{\epsilon}(x) f_{\epsilon}(y)}{|x-y|^{n-\alpha-\beta}} d x d y, \\
& I_{2}:=c^{2} \int_{B_{R}\left(x_{*}\right)} \int_{B_{R}\left(x_{*}\right)} \frac{\left|x-x_{*}\right|^{\gamma}\left|y-x_{*}\right|^{\gamma} f_{\epsilon}(x) f_{\epsilon}(y)}{|x-y|^{n-\alpha}} d x d y, \\
& I_{3}:=2 c \int_{B_{R}\left(x_{*}\right)} \int_{B_{R}\left(x_{*}\right)} \frac{K\left(x_{*}\right)\left|x-x_{*}\right|^{\gamma} f_{\epsilon}(x) f_{\epsilon}(y)}{|x-y|^{n-\alpha}} d x d y .
\end{aligned}
$$

For $I_{1}$, we have

$$
\begin{aligned}
I_{1} & =\lambda \int_{B_{R}\left(x_{*}\right)} \int_{B_{R}\left(x_{*}\right)} \frac{G(x) G(y)}{|x-y|^{n-\alpha-\beta}}\left(\frac{\epsilon}{\epsilon^{2}+\left|x-x_{*}\right|^{2}}\right)^{\frac{n+\alpha}{2}}\left(\frac{\epsilon}{\epsilon^{2}+\left|y-x_{*}\right|^{2}}\right)^{\frac{n+\alpha}{2}} d x d y \\
& \leq C \lambda \epsilon^{-(n-\alpha-\beta)-(n+\alpha)} \int_{B_{R}(0)} \int_{B_{R}(0)}\left|\frac{x-y}{\epsilon}\right|^{-(n-\alpha-\beta)}\left(1+\left|\frac{x}{\epsilon}\right|^{2}\right)^{-\frac{n+\alpha}{2}}\left(1+\left|\frac{y}{\epsilon}\right|^{2}\right)^{-\frac{n+\alpha}{2}} d x d y \\
& =C \lambda \epsilon^{\beta} \int_{B_{\frac{R}{\epsilon}(0)}} \int_{B_{\frac{R}{\epsilon}}(0)}|\xi-\eta|^{-(n-\alpha-\beta)}\left(1+|\xi|^{2}\right)^{-\frac{n+\alpha}{2}}\left(1+|\eta|^{2}\right)^{-\frac{n+\alpha}{2}} d \xi d \eta \\
& \leq C_{1} \lambda \epsilon^{\beta} .
\end{aligned}
$$

For $I_{2}$, we have

$$
\begin{aligned}
I_{2} & :=c^{2} \int_{B_{R}\left(x_{*}\right)} \int_{B_{R}\left(x_{*}\right)} \frac{\left|x-x_{*}\right|^{\gamma}\left|y-x_{*}\right|^{\gamma} f_{\epsilon}(x) f_{\epsilon}(y)}{|x-y|^{n-\alpha}} d x d y \\
& \leq c^{2} R^{2 \gamma} \int_{B_{R}\left(x_{*}\right)} \int_{B_{R}\left(x_{*}\right)} \frac{f_{\epsilon}(x) f_{\epsilon}(y)}{|x-y|^{n-\alpha}} d x d y \\
& \leq c^{2} R^{2 \gamma} \int_{\mathbb{R}^{n}} \int_{\mathbb{R}^{n}} \frac{f_{\epsilon}(x) f_{\epsilon}(y)}{|x-y|^{n-\alpha}} d x d y \\
& =C_{2} R^{2 \gamma}
\end{aligned}
$$

where $C_{2}:=c^{2} N_{\alpha}\left\|f_{\epsilon}\right\|_{L^{q \alpha}\left(\mathbb{R}^{n}\right)}^{2}$. For $I_{3}$, we have

$$
\begin{aligned}
I_{3} & :=2 c \int_{B_{R}\left(x_{*}\right)} \int_{B_{R}\left(x_{*}\right)} \frac{K\left(x_{*}\right)\left|x-x_{*}\right|^{\gamma} f_{\epsilon}(x) f_{\epsilon}(y)}{|x-y|^{n-\alpha}} d x d y \\
& \leq 2 c R^{\gamma} \int_{B_{R}\left(x_{*}\right)} \int_{B_{R}\left(x_{*}\right)} \frac{K\left(x_{*}\right) f_{\epsilon}(x) f_{\epsilon}(y)}{|x-y|^{n-\alpha}} d x d y \\
& \leq 2 c R^{\gamma} \int_{\mathbb{R}^{n}} \int_{\mathbb{R}^{n}} \frac{K\left(x_{*}\right) f_{\epsilon}(x) f_{\epsilon}(y)}{|x-y|^{n-\alpha}} d x d y \\
& =C_{3} R^{\gamma},
\end{aligned}
$$


where $C_{3}:=2 c K\left(x_{*}\right) N_{\alpha}\left\|f_{\epsilon}\right\|_{L^{q_{\alpha}\left(\mathbb{R}^{n}\right)}}^{2}$. Therefore, for $\lambda<0$, we can take $s$ satisfying $\frac{\beta}{\gamma}<s$, and $R=\epsilon^{s}>0$ for some $\epsilon>0$ small enough, such that

$$
\begin{aligned}
I_{1}+I_{2}+I_{3} & \leq C_{1} \lambda \epsilon^{\beta}+C_{2} R^{2 \gamma}+C_{3} R^{\gamma} \\
& =C_{1} \lambda \epsilon^{\beta}+C_{2} \epsilon^{2 s \gamma}+C_{3} \epsilon^{s \gamma}<0 .
\end{aligned}
$$

Combining this with (3.2), for $\lambda<0, \epsilon>0$ small enough, we have

$$
\int_{\Omega} \int_{\Omega}\left(\frac{K(x) K(y)}{|x-y|^{n-\alpha}}+\frac{\lambda G(x) G(y)}{|x-y|^{n-\alpha-\beta}}\right) \tilde{f}_{\epsilon}(x) \tilde{f}_{\epsilon}(y) d x d y<K^{2}\left(x_{*}\right) N_{\alpha}\left\|f_{\epsilon}\right\|_{L^{q_{\alpha}\left(\mathbb{R}^{n}\right)}}^{2} .
$$

That is, for any $\lambda<0, Q_{\lambda, q_{\alpha}}(\Omega)<K^{2}\left(x_{*}\right) N_{\alpha}$.

(II) Let $x_{*} \in \partial \Omega$. By $(\mathcal{T})$, there exists $\rho_{1}>0$ such that $K(x)-K\left(x_{*}\right) \leq c\left|x-x_{*}\right|^{\gamma}$ when $x \in V:=\bar{\Omega} \cap \overline{B\left(x_{*}, \rho_{1}\right)}$.

Let $0<\rho_{0}<\rho_{1}, x_{0} \in V$ satisfying $B_{\rho_{0}}\left(x_{0}\right) \subset V-\partial V,\left|x_{0}-x_{*}\right|=2 \rho_{0}$. Then, for any $x \in$ $B_{\rho_{0}}\left(x_{0}\right)$, we have

$$
K(x)-K\left(x_{*}\right) \leq C\left(\left|x-x_{0}\right|^{\gamma}+\left|x_{0}-x_{*}\right|^{\gamma}\right) .
$$

We define

$$
\tilde{f}_{\epsilon}(x)= \begin{cases}\bar{f}_{\epsilon}(x), & x \in B_{\rho_{0}}\left(x_{0}\right) \subset \Omega, \\ 0, & x \in \mathbb{R}^{n} \backslash B_{\rho_{0}}\left(x_{0}\right),\end{cases}
$$

where $\bar{f}_{\epsilon}(x)=\epsilon^{-\frac{n+\alpha}{2}} f_{1}\left(\frac{\left|x-x_{0}\right|}{\epsilon}\right)=\left(\frac{\epsilon}{\epsilon^{2}+\left|x-x_{0}\right|^{2}}\right)^{\frac{n+\alpha}{2}}$.

Similar to (I),

$$
\begin{aligned}
\int_{\Omega} & \int_{\Omega}\left(\frac{K(x) K(y)}{|x-y|^{n-\alpha}}+\frac{\lambda G(x) G(y)}{|x-y|^{n-\alpha-\beta}}\right) \tilde{f}_{\epsilon}(x) \tilde{f}_{\epsilon}(y) d x d y \\
= & \int_{B_{\rho_{0}}\left(x_{0}\right)} \int_{B_{\rho_{0}}\left(x_{0}\right)}\left(\frac{K(x) K(y)}{|x-y|^{n-\alpha}}+\frac{\lambda G(x) G(y)}{|x-y|^{n-\alpha-\beta}}\right) \bar{f}_{\epsilon}(x) \bar{f}_{\epsilon}(y) d x d y \\
\leq & \int_{B_{\rho_{0}}\left(x_{0}\right)} \int_{B_{\rho_{0}}\left(x_{0}\right)} \frac{\left(K\left(x_{*}\right)+C\left(\left|x-x_{0}\right|^{\gamma}+\left|2 \rho_{0}\right|^{\gamma}\right)\right)\left(K\left(x_{*}\right)+C\left(\left|y-x_{0}\right|^{\gamma}+\left|2 \rho_{0}\right|^{\gamma}\right)\right)}{|x-y|^{n-\alpha}} \\
& \times \bar{f}_{\epsilon}(x) \bar{f}_{\epsilon}(y) d x d y \\
& +\lambda \int_{B_{\rho_{0}}\left(x_{0}\right)} \int_{B_{\rho_{0}}\left(x_{0}\right)} \frac{G(x) G(y) \bar{f}_{\epsilon}(x) \bar{f}_{\epsilon}(y)}{|x-y|^{n-\alpha-\beta}} d x d y \\
\leq & \int_{\mathbb{R}^{n}} \int_{\mathbb{R}^{n}} \frac{K^{2}\left(x_{*}\right) \bar{f}_{\epsilon}(x) \bar{f}_{\epsilon}(y)}{|x-y|^{n-\alpha}} d x d y+\lambda \int_{B_{\rho_{0}}\left(x_{0}\right)} \int_{B_{\rho_{0}}\left(x_{0}\right)} \frac{G(x) G(y) \bar{f}_{\epsilon}(x) \bar{f}_{\epsilon}(y)}{|x-y|^{n-\alpha-\beta}} d x d y \\
& +2 C \int_{B_{\rho_{0}}\left(x_{0}\right)} \int_{B_{\rho_{0}}\left(x_{0}\right)} \frac{K\left(x_{*}\right)\left(\left|x-x_{0}\right|^{\gamma}+\left|2 \rho_{0}\right|^{\gamma}\right) \bar{f}_{\epsilon}(x) \bar{f}_{\epsilon}(y)}{|x-y|^{n-\alpha}} d x d y \\
& +C^{2} \int_{B_{\rho_{0}}\left(x_{0}\right)} \int_{B_{\rho_{0}}\left(x_{0}\right)} \frac{\left(\left|x-x_{0}\right|^{\gamma}+\left|2 \rho_{0}\right|^{\gamma}\right)\left(\left|y-x_{0}\right|^{\gamma}+\left|2 \rho_{0}\right|^{\gamma}\right) \bar{f}_{\epsilon}(x) \bar{f}_{\epsilon}(y)}{|x-y|^{n-\alpha}} d x d y \\
= & K^{2}\left(x_{*}\right) N_{\alpha}\left\|\bar{f}_{\epsilon}\right\|_{L^{q \alpha}\left(\mathbb{R}^{n}\right)}^{2}+J_{1}+J_{2}+J_{3},
\end{aligned}
$$


where

$$
\begin{aligned}
& J_{1}:=\lambda \int_{B_{\rho_{0}}\left(x_{0}\right)} \int_{B_{\rho_{0}}\left(x_{0}\right)} \frac{G(x) G(y) \bar{f}_{\epsilon}(x) \bar{f}_{\epsilon}(y)}{|x-y|^{n-\alpha-\beta}} d x d y, \\
& J_{2}:=2 C \int_{B_{\rho_{0}\left(x_{0}\right)}} \int_{B_{\rho_{0}\left(x_{0}\right)}} \frac{K\left(x_{*}\right)\left(\left|x-x_{0}\right|^{\gamma}+\left|2 \rho_{0}\right|^{\gamma}\right) \bar{f}_{\epsilon}(x) \bar{f}_{\epsilon}(y)}{|x-y|^{n-\alpha}} d x d y, \\
& J_{3}:=C^{2} \int_{B_{\rho_{0}}\left(x_{0}\right)} \int_{B_{\rho_{0}}\left(x_{0}\right)} \frac{\left(\left|x-x_{0}\right|^{\gamma}+\left|2 \rho_{0}\right|^{\gamma}\right)\left(\left|y-x_{0}\right|^{\gamma}+\left|2 \rho_{0}\right|^{\gamma}\right) \bar{f}_{\epsilon}(x) \bar{f}_{\epsilon}(y)}{|x-y|^{n-\alpha}} d x d y .
\end{aligned}
$$

As in case (I), we know $J_{1} \leq C_{4} \lambda \epsilon^{\beta}$. For $J_{2}$, we have

$$
\begin{aligned}
J_{2} & =2 C \int_{B_{\rho_{0}}\left(x_{0}\right)} \int_{B_{\rho_{0}\left(x_{0}\right)}} \frac{K\left(x_{*}\right)\left(\left|x-x_{0}\right|^{\gamma}+\left|2 \rho_{0}\right|^{\gamma}\right) \bar{f}_{\epsilon}(x) \bar{f}_{\epsilon}(y)}{|x-y|^{n-\alpha}} d x d y \\
& \leq C K\left(x_{*}\right) \rho_{0}^{\gamma} \int_{B_{\rho_{0}\left(x_{0}\right)}} \int_{B_{\rho_{0}\left(x_{0}\right)}} \frac{\bar{f}_{\epsilon}(x) \bar{f}_{\epsilon}(y)}{|x-y|^{n-\alpha}} d x d y \\
& \leq C_{5} \rho_{0}^{\gamma} .
\end{aligned}
$$

For $J_{3}$, we have

$$
\begin{aligned}
J_{3} & =C^{2} \int_{B_{\rho_{0}}\left(x_{0}\right)} \int_{B_{\rho_{0}\left(x_{0}\right)}} \frac{\left(\left|x-x_{0}\right|^{\gamma}+\left|2 \rho_{0}\right|^{\gamma}\right)\left(\left|y-x_{0}\right|^{\gamma}+\left|2 \rho_{0}\right|^{\gamma}\right) \bar{f}_{\epsilon}(x) \bar{f}_{\epsilon}(y)}{|x-y|^{n-\alpha}} d x d y \\
& \leq C^{2} \rho_{0}^{2 \gamma} \int_{B_{\rho_{0}\left(x_{0}\right)}} \int_{B_{\rho_{0}}\left(x_{0}\right)} \frac{\bar{f}_{\epsilon}(x) \bar{f}_{\epsilon}(y)}{|x-y|^{n-\alpha}} d x d y \\
& \leq C_{6} \rho_{0}^{2 \gamma} .
\end{aligned}
$$

Taking $s$ with $\frac{\beta}{\gamma}<s$ and $\rho_{0}=\epsilon^{s}>0$, then

$$
\begin{aligned}
J_{1}+J_{2}+J_{3} & \leq C_{4} \lambda \epsilon^{\beta}+C_{5} \rho_{0}^{\gamma}+C_{6} \rho_{0}^{2 \gamma} \\
& =C_{4} \lambda \epsilon^{\beta}+C_{5} \epsilon^{s \gamma}+C_{6} \epsilon^{2 s \gamma}<0
\end{aligned}
$$

for $\epsilon>0$ small enough. Thus, combining this with (3.3), for $\lambda<0, \epsilon>0$ small enough, we have

$$
\int_{\Omega} \int_{\Omega}\left(\frac{K(x) K(y)}{|x-y|^{n-\alpha}}+\frac{\lambda G(x) G(y)}{|x-y|^{n-\alpha-\beta}}\right) \tilde{f}_{\epsilon}(x) \tilde{f}_{\epsilon}(y) d x d y<K^{2}\left(x_{*}\right) N_{\alpha}\left\|\bar{f}_{\epsilon}\right\|_{L^{q_{\alpha}}\left(\mathbb{R}^{n}\right)}^{2} .
$$

That is, for any $\lambda<0$, we have $Q_{\lambda, q_{\alpha}}(\Omega)<K^{2}\left(x_{*}\right) N_{\alpha}$.

On the other hand, for any $\lambda \in\left(-\frac{K^{2}\left(x_{*}\right)}{d^{\beta}(\Omega) G^{2}\left(\tilde{x}_{*}\right)}, 0\right)$, we also have $Q_{\lambda, q_{\alpha}}(\Omega)>0$. So we complete the proof.

In order to prove the existence of weak solutions, we need to prove that the minimal energy $Q_{\lambda, q_{\alpha}}(\Omega)$ is attained.

Proposition 3.5 For given $\lambda \in\left(-\frac{K^{2}\left(x_{*}\right)}{d^{\beta}(\Omega) G^{2}\left(\tilde{x}_{*}\right)}, 0\right), Q_{\lambda, q_{\alpha}}(\Omega)$ is attained by some positive function $f_{*} \in L^{q_{\alpha}}(\Omega)$. 
For $q<q_{\alpha}$, we consider

$$
\begin{aligned}
& Q_{\lambda, q}(\Omega) \\
& \quad=\inf _{f \in L_{+}^{q}(\Omega)} \frac{\int_{\Omega} \int_{\Omega} f(x)\left(K(x)|x-y|^{-(n-\alpha)} K(y)+\lambda G(x)|x-y|^{-(n-\alpha-\beta)} G(y)\right) f(y) d x d y}{\|f\|_{L^{q}(\Omega)}^{2}} .
\end{aligned}
$$

By Lemma 3.2, the infimum is attained by the positive function $f_{q}$ which satisfies the integral equation with the subcritical exponent

$$
Q_{\lambda, q}(\Omega) f^{q-1}(x)=\int_{\Omega} \frac{K(x) f(y) K(y)}{|x-y|^{n-\alpha}} d y+\lambda \int_{\Omega} \frac{G(x) f(y) G(y)}{|x-y|^{n-\alpha-\beta}} d y, \quad x \in \bar{\Omega} .
$$

That is, $f_{q}$ is the minimal energy solution to Eq. (3.4). It is easy to see that $\left\|f_{q}\right\|_{L^{q}(\Omega)}=1$, $f_{q} \in C(\bar{\Omega})$ and $Q_{\lambda, q} \rightarrow Q_{\lambda}$ as $q \rightarrow\left(q_{\alpha}\right)^{-}$.

Lemma 3.6 For $\lambda \in\left(-\frac{K^{2}\left(x_{*}\right)}{d^{\beta}(\Omega) G^{2}\left(\tilde{x}_{*}\right)}, 0\right), q \in\left(0, q_{\alpha}\right)$. Let $f_{q}>0$ be a minimal energy solution to (3.4), where $\left\|f_{q}\right\|_{L^{q}(\Omega)}=1$. If $0<Q_{\lambda, q}(\Omega) \leq K^{2}\left(x_{*}\right) N_{\alpha}-\epsilon$ for some $\epsilon>0$, then there exists $C>0$ such that $\frac{1}{C} \leq f_{q}(x) \leq C$ uniformly for all $x \in \bar{\Omega}, q \in\left(0, q_{\alpha}\right)$.

Proof We prove it by modifying the argument of Lemma 4.3 in [1].

For any $x \in \bar{\Omega}, q \in\left(0, q_{1}\right)$, we see that $\max _{\bar{\Omega}} f_{q}(x)=f_{q}\left(x_{q}\right) \leq C<\infty$ holds uniformly, where $0<q_{1}<q_{\alpha}$.

We first prove that $\max _{\bar{\Omega}} f_{q}(x)=f_{q}\left(x_{q}\right) \leq C<\infty$ holds uniformly as $q \rightarrow\left(q_{\alpha}\right)^{-}$. Otherwise, $f_{q}\left(x_{q}\right) \rightarrow \infty$, where $x_{q} \rightarrow \tilde{x}$, up to a subsequence, as $q \rightarrow\left(q_{\alpha}\right)^{-}$. Denote

$$
\mu_{q}:=f_{q}^{-\frac{2-q}{\alpha}}\left(x_{q}\right), \quad \Omega_{\mu}:=\frac{\Omega-x_{q}}{\mu_{q}}=\left\{z \mid z=\frac{x-x_{q}}{\mu_{q}}, x \in \Omega\right\} .
$$

We define

$$
g_{q}(z)=\mu_{q}^{\frac{\alpha}{2-q}} f_{q}\left(\mu_{q} z+x_{q}\right), \quad z \in \bar{\Omega}_{\mu}
$$

Thus $g_{q}$ satisfies

$$
\begin{aligned}
Q_{\lambda, q}(\Omega) g_{q}^{q-1}(z)= & \int_{\Omega_{\mu}} \frac{K\left(\mu_{q} z+x_{q}\right) g_{q}(y) K\left(\mu_{q} y+x_{q}\right)}{|z-y|^{n-\alpha}} d y \\
& +\lambda \mu_{q}^{\beta} \int_{\Omega_{\mu}} \frac{G\left(\mu_{q} z+x_{q}\right) g_{q}(y) G\left(\mu_{q} y+x_{q}\right)}{|z-y|^{n-\alpha-\beta}} d y, \quad z \in \bar{\Omega}_{\mu},
\end{aligned}
$$

and $g_{q}(0)=1, g_{q}(z) \in(0,1]$.

For convenience, we define $h_{q}(z):=g_{q}^{q-1}(z)$. So (3.5) is equivalent to

$$
\begin{aligned}
Q_{\lambda, q}(\Omega) h_{q}(z)= & \int_{\Omega_{\mu}} \frac{K\left(\mu_{q} z+x_{q}\right) h_{q}^{p-1}(y) K\left(\mu_{q} y+x_{q}\right)}{|z-y|^{n-\alpha}} d y \\
& +\lambda \mu_{q}^{\beta} \int_{\Omega_{\mu}} \frac{G\left(\mu_{q} z+x_{q}\right) h_{q}^{p-1}(y) G\left(\mu_{q} y+x_{q}\right)}{|z-y|^{n-\alpha-\beta}} d y, \quad z \in \bar{\Omega}_{\mu},
\end{aligned}
$$

where $\frac{1}{p}+\frac{1}{q}=1, h_{q}(0)=1, h_{q}(z) \geq 1$. 
Claim: There exist $C_{1}, C_{2}>0$ such that

$$
0<C_{1}\left(1+|z|^{\alpha-n}\right) \leq h_{q}(z) \leq C_{2}\left(1+|z|^{\alpha-n}\right), z \in \tilde{\Omega},
$$

holds uniformly for any domain $\tilde{\Omega} \subset \Omega_{\mu}$ as $q \rightarrow\left(q_{\alpha}\right)^{-}$.

The claim can be verified by a similar argument to that in [1], we omit it here. Thus $h_{q}(z)$ is equicontinuous in any bounded domain $\widehat{\Omega} \subset \Omega_{\mu}$ as $q \rightarrow\left(q_{\alpha}\right)^{-}$. In fact, for $R>0$,

$$
\begin{aligned}
& Q_{\lambda, q}(\Omega) h_{q}(z) \\
& =\int_{\Omega_{\mu} \backslash B(0, R)} \frac{K\left(\mu_{q} z+x_{q}\right) h_{q}^{p-1}(y) K\left(\mu_{q} y+x_{q}\right)}{|z-y|^{n-\alpha}} d y \\
& \quad+\int_{\Omega_{\mu} \cap B(0, R)} \frac{K\left(\mu_{q} z+x_{q}\right) h_{q}^{p-1}(y) K\left(\mu_{q} y+x_{q}\right)}{|z-y|^{n-\alpha}} d y \\
& \quad+\lambda \mu_{q}^{\beta} \int_{\Omega_{\mu} \backslash B(0, R)} \frac{G\left(\mu_{q} z+x_{q}\right) h_{q}^{p-1}(y) G\left(\mu_{q} y+x_{q}\right)}{|z-y|^{n-\alpha-\beta}} d y \\
& \quad+\lambda \mu_{q}^{\beta} \int_{\Omega_{\mu} \cap B(0, R)} \frac{G\left(\mu_{q} z+x_{q}\right) h_{q}^{p-1}(y) G\left(\mu_{q} y+x_{q}\right)}{|z-y|^{n-\alpha-\beta}} d y .
\end{aligned}
$$

Notice that

$$
\begin{aligned}
& \int_{\Omega_{\mu} \backslash B(0, R)} \frac{h_{q}^{p-1}(y)}{|z-y|^{n-\alpha}}\left(K\left(\mu_{q} z+x_{q}\right) K\left(\mu_{q} y+x_{q}\right)+\lambda \mu_{q}^{\beta} G\left(\mu_{q} z+x_{q}\right) G\left(\mu_{q} y+x_{q}\right)|z-y|^{\beta}\right) d y \\
& \quad \geq\left(K^{2}\left(x_{*}\right)-|\lambda| d^{\beta}(\Omega) G^{2}\left(\tilde{x}_{*}\right)\right) \int_{\Omega_{\mu} \backslash B(0, R)} \frac{h_{q}^{p-1}(y)}{|z-y|^{n-\alpha}} d y \geq 0 .
\end{aligned}
$$

For any $\epsilon>0$ small enough, by (3.6), we have

$$
\begin{aligned}
0 \leq & \int_{\Omega_{\mu} \backslash B(0, R)} \frac{h_{q}^{p-1}(y)}{|z-y|^{n-\alpha}}\left(K\left(\mu_{q} z+x_{q}\right) K\left(\mu_{q} y+x_{q}\right)\right. \\
& \left.+\lambda \mu_{q}^{\beta} G\left(\mu_{q} z+x_{q}\right)|z-y|^{\beta} G\left(\mu_{q} y+x_{q}\right)\right) d y \\
\leq & C \int_{\Omega_{\mu} \backslash B(0, R)} \frac{h_{q}^{p-1}(y)}{|y|^{n-\alpha}} d y \\
\leq & C \int_{R}^{\infty} r^{(\alpha-n)(p-1)+\alpha-1} d r \\
= & C R^{(\alpha-n)(p-1)+\alpha}<\epsilon,
\end{aligned}
$$

where $R>0$ is large enough, $q \rightarrow\left(q_{\alpha}\right)^{-}, z \in \widehat{\Omega}$. Since $\beta<n$, by using the same argument as above, we also have

$$
\left|\lambda \mu_{q}^{\beta} \int_{\Omega_{\mu} \cap B(0, R)} \frac{G\left(\mu_{q} z+x_{q}\right) h_{q}^{p-1}(y) G\left(\mu_{q} y+x_{q}\right)}{|z-y|^{n-\alpha-\beta}} d y\right|<\epsilon,
$$

when $R>0$ large enough, $q \rightarrow\left(q_{\alpha}\right)^{-}$. 
On the other hand, it is easy to see that, for $z \in \widehat{\Omega}, \int_{\Omega_{\mu} \cap B(0, R)} \frac{K\left(\mu_{q} z+x_{q}\right) h_{q}^{p-1}(y) K\left(\mu_{q} y+x_{q}\right)}{|z-y|^{n-\alpha}} d y \in$ $C^{1}(\widehat{\Omega})$. Combining this with (3.7) and (3.8), we conclude that $h_{q}(z)$ is equicontinuous in any bounded domain $\widehat{\Omega} \subset \mathbb{R}^{n}$ as $q \rightarrow\left(q_{\alpha}\right)^{-}$.

When $q \rightarrow\left(q_{\alpha}\right)^{-}$, we distinguish two cases:

Case 1. $\Omega_{\mu} \rightarrow \mathbb{R}_{T}^{n}:=\left\{\left(z_{1}, z_{2}, \ldots, z_{n}\right) \mid z_{n}>-T\right\}$ for some $T \geq 0$, and $h_{q}(z) \rightarrow h(z) \in C\left(\mathbb{R}_{T}^{n}\right)$ holds uniformly on any compact sets of $\mathbb{R}_{T}^{n}$, where $h(z)$ satisfying

$$
Q_{\lambda} h(z)=K^{2}(\widetilde{x}) \int_{\mathbb{R}_{T}^{n}} \frac{h^{p_{\alpha}-1}(y)}{|z-y|^{n-\alpha}} d y, \quad h(0)=1 .
$$

Then, similar to Lemma 4.3 in [1], we obtain a contradiction.

Case 2. $\Omega_{\mu} \rightarrow \mathbb{R}^{n}, h_{q}(z) \rightarrow h(z) \in C\left(\mathbb{R}^{n}\right)$ holds uniformly on any compact sets of $\mathbb{R}^{n}$, where $h(z)$ satisfying

$$
Q_{\lambda} h(z)=K^{2}(\widetilde{x}) \int_{\mathbb{R}^{n}} \frac{h^{p_{\alpha}-1}(y)}{|z-y|^{n-\alpha}} d y, \quad h(0)=1 .
$$

Again similar to Lemma 4.3 in [1], we obtain a contradiction.

Thus we conclude that there exists $C>0$ such that $f_{q}(y) \leq C$ uniformly for $y \in \bar{\Omega}, q \in$ $\left(0, q_{\alpha}\right)$.

On the other hand, if $\min _{\bar{\Omega}} f_{q}(x):=f_{q}\left(\widetilde{x}_{q}\right) \rightarrow 0$ as $q \rightarrow\left(q_{\alpha}\right)^{-}$. Then

$$
\infty \leftarrow f_{q}^{q-1}\left(\widetilde{x}_{q}\right)=\int_{\Omega} \frac{K\left(\widetilde{x}_{q}\right) f_{q}(y) K(y)}{\left|\widetilde{x}_{q}-y\right|^{n-\alpha}} d y+\lambda \int_{\Omega} \frac{G\left(\widetilde{x}_{q}\right) f_{q}(y) G(y)}{\left|\widetilde{x}_{q}-y\right|^{n-\alpha-\beta}} d y \leq C<\infty,
$$

as $q \rightarrow\left(q_{\alpha}\right)^{-}$, which implies a contradiction.

Proof of Proposition 3.5 By Lemma 3.6, $\left\{f_{q}\right\}$ are uniformly bounded above and bounded below by a positive constant. Thus the $\left\{f_{q}\right\}$ are equicontinuous due to Eq. (3.4). It follows that $f_{q} \rightarrow f_{*}$ as $q \rightarrow\left(q_{\alpha}\right)^{-}$in $C(\bar{\Omega})$, and $f_{*}$ is the energy minimizer for $Q_{\lambda}$.

Proof of Theorem 1.1 (ii) Lemma 3.4 and Proposition 3.5 imply the existence of a positive solution $f \in L^{q_{\alpha}}(\Omega) \cap C(\bar{\Omega})$ to Eq. (1.1) for $q=\frac{2 n}{n+\alpha}, \lambda \in\left(-\frac{K^{2}\left(x_{*}\right)}{d^{\beta}(\Omega) G^{2}\left(\tilde{x}_{*}\right)}, 0\right)$. It is also easy to see that $f \in C^{1}(\bar{\Omega})$.

\subsection{Nonexistence-critical and supercritical case}

We first state a Pohozaev type identity.

Lemma 3.7 Assume that the origin is in $\Omega$ and the domain is star-shaped with respect to the origin. If $u \in C^{1}(\bar{\Omega})$ is a nonnegative solution to

$$
u(x)=\int_{\Omega} \frac{K(x) u^{p-1}(y) K(y)}{|x-y|^{n-\alpha}} d y+\lambda \int_{\Omega} \frac{G(x) u^{p-1}(y) G(y)}{|x-y|^{n-\alpha-\beta}} d y, \quad x \in \bar{\Omega},
$$

where $p \neq 0, \lambda \in \mathbb{R}, K(x), G(x) \in C^{1}(\bar{\Omega})$, then

$$
\left(\frac{n}{p}+\frac{\alpha-n}{2}\right) \int_{\Omega} u^{p}(x) d x
$$




$$
\begin{aligned}
= & -\frac{\lambda \beta}{2} \int_{\Omega} \int_{\Omega} \frac{G(x) u^{p-1}(x) u^{p-1}(y) G(y)}{|x-y|^{n-\alpha-\beta}} d y d x+\frac{1}{p} \int_{\partial \Omega}(x \cdot v) u^{p}(x) d \sigma \\
& -\int_{\Omega} \int_{\Omega} \frac{(x, \nabla K(x)) u^{p-1}(x) u^{p-1}(y) K(y)}{|x-y|^{n-\alpha}} d y d x \\
& -\lambda \int_{\Omega} \int_{\Omega} \frac{(x, \nabla G(x)) u^{p-1}(x) u^{p-1}(y) G(y)}{|x-y|^{n-\alpha-\beta}} d y d x,
\end{aligned}
$$

where $v$ is the outward unit normal vector to $\partial \Omega$.

Proof The argument is standard. We omit it here.

Proof of Theorem 1.1(iii) We can prove by using Lemma 3.7 and a similar argument to that used in [1]. We omit it here.

\section{Acknowledgements}

The authors would like to thank the anonymous referee for very valuable suggestions and comments, and thank Dr. Jiankang Xia for many helpful discussions and valuable comments during the preparation of this manuscript.

\section{Funding}

The first author is supported by the Fundamental Research Funds for the Central Universities Grant No. 310201911 cx013. The second and the third authors are supported by the National Natural Science Foundation of China (Grant No. 11971385) and the Natural Science Basic Research Plan in Shaanxi Province of China (Grant No. 2019JM-275).

\section{Availability of data and materials}

Not applicable.

\section{Competing interests}

The authors declare that no competing interests exist.

\section{Authors' contributions}

All authors contributed equally. All authors read and approved the final manuscript.

\section{Publisher's Note}

Springer Nature remains neutral with regard to jurisdictional claims in published maps and institutional affiliations.

Received: 18 February 2020 Accepted: 17 April 2020 Published online: 29 April 2020

\section{References}

1. Dou, J., Guo, Q., Zhu, M.: Negative power nonlinear integral equations on bounded domains. arXiv:1904.03878 (2019)

2. Dou, J., Zhu, M.: Nonlinear integral equations on bounded domains. J. Funct. Anal. 277, 111-134 (2019)

3. Guo, Q., Wang, Q.: Existence of positive solutions to integral equations with weights. Appl. Math. Lett. 102, 106089 (2020)

4. Hardy, G.H., Littlewood, J.E.: Some properties of fractional integrals (1). Math. Z. 27, 565-606 (1928)

5. Hardy, G.H., Littlewood, J.E.: On certain inequalities connected with the calculus of variations. J. Lond. Math. Soc. 5, 34-39 (1930)

6. Lieb, E.H.: Sharp constants in the Hardy-Littlewood-Sobolev and related inequalities. Ann. Math. 118, 349-374 (1983)

7. Sobolev, S.L.: On a theorem of functional analysis. Mat. Sb. (N.S.) 4, 471-479 (1938). Transl. Am. Math. Soc. 34, 39-68 (1963)

8. Beckner, W.: Functionals for multilinear fractional embedding. Acta Math. Sin. Engl. Ser. 31, 1-28 (2015)

9. Dou, J., Zhu, M.: Reversed Hardy-Littlewood-Sobolev inequality. Int. Math. Res. Not. 2015, 9696-9726 (2015)

10. Guo, Q.: Blowup analysis for integral equations on bounded domains. J. Differ. Equ. 266, 8258-8280 (2019) 